\title{
Nuclear shadowing in the light-cone dipole approach
}

\section{Jan Nemchik*}

IEP SAS, Slovakia

E-mail: nemcik@saske.sk

We study nuclear shadowing at small Bjorken $x_{B j} \lesssim 0.01$ in the color dipole approach. Such a light-cone quantum-chromodynamics formalism based on the Green function technique incorporates naturally color transparency and coherence length effects. The nuclear shadowing for the $\bar{q} q$ Fock component of the photon is calculated using exact numerical solution of the evolution equation for the Green function. At $x_{B j} \leq 0.01$ we demonstrate that a contribution of higher Fock states containing gluons to overall nuclear shadowing becomes effective. Numerical results for nuclear shadowing are compared with available data from the E665 and NMC collaborations. Model calculations are finally tested with the results obtained from other models.

PACS: $13.85 . \mathrm{Lg}, 13.60 . \mathrm{Le}$

High-pT physics at LHC

March 23-27, 2007

University of Jyvaskyla, Jyvaskyla, Finland

\footnotetext{
*Speaker.
} 


\section{Introduction}

Nuclear shadowing (NS) in deep-inelastic scattering (DIS) off nuclei is usually studied via nuclear structure functions. Assuming shadowing region of small Bjorken $x_{B j} \lesssim 0.01$ the structure function $F_{2}$ per nucleon turns out to be smaller in nuclei than in a free nucleon. This fact affects then the corresponding study of nuclear effects mainly in connection with the interpretation of the results from hadron-nucleus and heavy ion experiments.

There are different treatments of NS depending on the reference frame. In the infinite momentum frame of the nucleus NS can be interpreted as a result of parton fusion [1, 2] leading to a reduction of the parton density at low Bjorken $x_{B j}$. In the rest frame of the nucleus, however, this phenomenon looks like NS of the virtual photon hadronic fluctuations and is occurred due to their multiple scattering inside the target (see refs. [3, 4, 5] and references therein, for example).

In the rest frame of the nucleus, the dynamics of NS is controlled by the time scale known as the effect of quantum coherence. It results from destructive interference of the amplitudes for which the interaction takes place on different bound nucleons. It can be estimated by relying on the uncertainty principle and Lorentz time dilation. For the lowest Fock component of the photon it reads,

$$
t_{c}=\frac{2 v}{Q^{2}+M_{\bar{q} q}^{2}}
$$

where $v$ and $Q^{2}$ is the photon energy and virtuality, respectively and $M_{\bar{q} q}$ is the effective mass of the ${ }^{-} q q$ pair. It is usually called coherence time, but we also will use the term coherence length (CL), since light-cone (LC) kinematics is assumed, $l_{c}=t_{c}$. CL is related to the longitudinal momentum transfer $q_{c}=1 / l_{c}$.

Note, that higher Fock states containing gluons, $\left|{ }^{-} q q G\right\rangle,\left|{ }^{-} q q 2 G\right\rangle, \ldots$ have larger effective masses than ${ }^{-} q q$ fluctuation resulting in shorter coherence times.

The Green function approach [6] is a very effective tool for the study of NS naturally incorporating the effects of CL. Such a formalism is based on the solution of the evolution equation for the Green function. Usually, one has been forced to obtain an analytical solution leading to a harmonic oscillator form (see Eq. (2.11). It requires, however, to implement several approximations into a rigorous quantum-mechanical approach like a constant nuclear density function (2.19) and a specific quadratic form (2.18) of the dipole cross section.

We remove such approximations for the $\left|{ }^{-} q q\right\rangle$ Fock state solving the evolution equation numerically using algorithm from ref. [5]. It is supported also by the fact that the predictions for NS [5] corresponding to the lowest Fock component of the photon showed quite large differences from approximate calculations [7, 虰 using the harmonic oscillator Green function approach for $l_{c} \lesssim R_{A}$ ( $R_{A}$ is the nuclear radius).

Calculations of NS presented so far in the color dipole approach [7, 4, 5] were performed assuming only ${ }^{-} q q$ fluctuations of the photon and neglecting higher Fock components containing gluons and sea quarks. The effects of higher Fock states are included in energy dependence of the dipole cross section, $\sigma_{\bar{q} q}(\vec{r}, s)^{1}$. However, investigating nuclear effects, these Fock states lead also to gluon shadowing (GS), which has been neglected so far when the model predictions were compared with experimental data.

\footnotetext{
${ }^{1}$ Here $\vec{r}$ represents the transverse separation of the $\bar{q} q$ photon fluctuation and $s$ is the center of mass energy squared.
} 
It was demonstrated in ref. [6] that a contribution of GS to overall NS becomes effective at small $x_{B j} \lesssim 0.01$. It confirms the necessity for inclusion of GS in the model predictions in the shadowing region $\sim 0.0001 \lesssim x_{B j} \lesssim 0.01$, where the data exist.

\section{Light-cone dipole approach to nuclear shadowing}

In the rest frame of the nucleus NS in the total virtual photoabsorption cross section $\sigma_{\text {tot }}^{\gamma^{*} A}\left(x_{B j}, Q^{2}\right)$ (or in the structure function $F_{2}^{A}\left(x_{B j}, Q^{2}\right)$ ) can be decomposed over different Fock components of the virtual photon. Then the total photoabsorption cross section on a nucleus can be formally represented in the form

$$
\sigma_{\text {tot }}^{\gamma^{*} A}\left(x_{B j}, Q^{2}\right)=A \sigma_{t o t}^{\gamma^{*} N}\left(x_{B j}, Q^{2}\right)-\Delta \sigma_{t o t}\left(x_{B j}, Q^{2}\right),
$$

where $\Delta \sigma_{t o t}\left(x_{B j}, Q^{2}\right)=\Delta \sigma_{t o t}\left({ }^{-} q q\right)+\Delta \phi_{o t}\left({ }^{-} q q G\right)+\Delta \phi_{o t}\left({ }^{-} q q 2 G\right)+\ldots,{ }_{B j}$ is the Bjorken variable and $\sigma_{\text {tot }}^{\gamma^{*} N}\left(x_{B j}, Q^{2}\right)$ is total photoabsorption cross section on a nucleon

$$
\sigma_{t o t}^{\gamma^{*} N}\left(x_{B j}, Q^{2}\right)=\int d^{2} r \int_{0}^{1} d \alpha\left|\Psi_{\bar{q} q}\left(\vec{r}, \alpha, Q^{2}\right)\right|^{2} \sigma_{\bar{q} q}(\vec{r}, s) .
$$

The dipole cross section $\sigma_{\bar{q} q}(\vec{r}, s)$ in Eq. (2.2) represents the interaction of a ${ }^{-} q q$ dipole of transverse separation $\vec{r}$ with a nucleon [8]. It depends on the c.m. energy squared $s$. The dependence of $\sigma_{\bar{q} q}(\vec{r}, s)$ on $\vec{r}$ and energy is universal and flavor independent allowing to describe various high energy processes in an uniform way. $\sigma_{\bar{q} q}(\vec{r}, s)$ is known to vanish quadratically $\propto r^{2}$ as $r \rightarrow 0$ due to color screening (property of color transparency [8, 9]). It cannot be predicted reliably because of poorly known higher order perturbative QCD (pQCD) corrections and nonperturbative effects. However, $\sigma_{\bar{q} q}(\vec{r}, s)$ can be extracted from experimental data on DIS and structure functions using reasonable parametrizations. In this case $\mathrm{pQCD}$ corrections and nonperturbative effects are naturally included.

There are two popular parametrizations of $\sigma_{\bar{q} q}(\vec{r}, s)$, GBW presented in [10] and KST suggested in [6]. Detailed discussion and comparison of these two parametrizations can be found in refs. [11, 5]. Whereas GBW parametrization can not be applied in the nonperturbative region of small $Q^{2}$, KST parametrization presents a good description of the transition down to limit of real photoproduction, $Q^{2}=0$. Because we study the shadowing region of small $x_{B j} \lesssim 0.01$ and available experimental data from the E665 and NMC collaborations cover small and moderate values of $Q^{2} \lesssim 2 \div 3 \mathrm{GeV}^{2}$ we prefer the latter parametrization.

The KST parametrization [6] has the following form containing an explicit energy dependence,

$$
\sigma_{\bar{q} q}(r, s)=\sigma_{0}(s)\left[1-\exp \left(-\frac{r^{2}}{R_{0}^{2}(s)}\right)\right]
$$

where

$$
\sigma_{0}(s)=\sigma_{t o t}^{\pi p}(s)\left(1+\frac{3 R_{0}^{2}(s)}{8\left\langle r_{c h}^{2}\right\rangle_{\pi}}\right) .
$$

In Eq. (2.3) the energy-dependent radius $R_{0}(s)=0.88\left(s / s_{0}\right)^{-\lambda / 2} \mathrm{fm}$ with $\lambda=0.28$ and $s_{0}=$ $1000 \mathrm{GeV}^{2}$. In Eq. (2.4) $\sigma_{\text {tot }}^{\pi p}(s)=23.6\left(s / s_{0}\right)^{0.079}+1.432\left(s / s_{0}\right)^{-0.45} \mathrm{mb}$ corresponding to the 
Pomeron and Reggeon parts of the $\pi p$ total cross section [12] and $\left\langle r_{c h}^{2}\right\rangle_{\pi}=0.44 \mathrm{fm}^{2}$ represents the mean pion charge radius squared.

Several comments about KST parametrization are in order:

i) The KST dipole cross section (2.3) is proportional to $r^{2}$ for $r \rightarrow 0$, but flattens off at large $r$.

ii) The saturation scale $R_{0}(s)$ decreases with increasing $s$. The energy dependence of $\sigma_{\bar{q} q}(r, s)$ correlates with $r$. At small $r$ the dipole cross section rises with a hard pomeron intercept, 0.36 , and at large separations it still depends with a soft pomeron intercept, 0.08 , on energy.

iii) In contrast to GBW parametrization [10], energy dependent ansatz, Eq. (2.3), allows to describe simultaneously hadron-hadron scattering and DIS. This improvement at large separations leads to a worse description of the short-distance part of the dipole cross section which is responsible for the behavior of $F_{2}^{p}\left(x_{B j}, Q^{2}\right)$ at large $Q^{2}$. To satisfy Bjorken scaling the dipole cross section at small $r$ must be a function of the product $s r$ which is not the case for the parametrization in Eq. (2.3).

iv) The form of Eq. (2.3) successfully describes the data for DIS at small $x_{B j}$ only up to $Q^{2} \approx 10 \mathrm{GeV}^{2}$. Nevertheless, this interval of $Q^{2}$ is sufficient for the purpose of the present paper.

The second ingredient, $\Psi_{\bar{q} q}\left(\vec{r}, \alpha, Q^{2}\right)$, in (2.2) is the perturbative distribution amplitude ("wave function") of the - $q q$ Fock component of the photon. It depends on the photon virtuality $\mathcal{Q}$ and the relative share $\alpha$ of the photon momentum carried by the quark. The corresponding form for transversely (T) and longitudinally (L) polarized photons can be found in refs. [13, 14, 3]:

$$
\Psi_{\bar{q} q}^{T, L}\left(\vec{r}, \alpha, Q^{2}\right)=\frac{\sqrt{N_{C} \alpha_{e m}}}{2 \pi} Z_{q} \bar{\chi} \hat{O}^{T, L} \chi K_{0}(\varepsilon r)
$$

where $\chi$ and $\bar{\chi}$ are the spinors of the quark and antiquark, respectively; $Z_{q}$ is the quark charge, $N_{C}=3$ is the number of colors. $K_{0}(\varepsilon r)$ is a modified Bessel function with

$$
\varepsilon^{2}=\alpha(1-\alpha) Q^{2}+m_{q}^{2},
$$

where $m_{q}$ is the quark mass. The form of the operators $\hat{O}^{T, L}$ can be found in [6].

The distribution amplitude Eq. (2.5) controls the transverse ${ }^{-} q q$ separation with the mean value,

$$
\langle r\rangle \sim \frac{1}{\varepsilon}=\frac{1}{\sqrt{Q^{2} \alpha(1-\alpha)+m_{q}^{2}}} .
$$

For very asymmetric ${ }^{-} q q$ pairs with $\alpha$ or $(1-\alpha) \lesssim m_{q}^{2} / Q^{2}$ the mean transverse separation $\langle r\rangle \sim 1 / m_{q}$ becomes huge since one must use current quark masses within $\mathrm{pQCD}$. A popular recipe to fix this problem is to introduce an effective quark mass $m_{\text {eff }} \sim \Lambda_{Q C D}$ which should represent the nonperturbative interaction effects between $q$ and ${ }^{-} q$. However, we introduce this interaction explicitly using corresponding phenomenology based on the LC Green function approach developed in [6].

The Green function $G_{\bar{q} q}\left(\vec{r}_{2}, z_{2} ; \vec{r}_{1}, z_{1}\right)$ describes the propagation of an interacting $q q$ pair between points with longitudinal coordinates $z_{1}$ and $z_{2}$ and with initial and final separations $\vec{r}_{1}$ and $\vec{r}_{2}$. This Green function satisfies the two-dimensional Schrödinger equation,

$$
i \frac{d}{d z_{2}} G_{\bar{q} q}\left(\vec{r}_{2}, z_{2} ; \vec{r}_{1}, z_{1}\right)=\left[\frac{\varepsilon^{2}-\Delta_{r_{2}}}{2 v \alpha(1-\alpha)}+V_{\bar{q} q}\left(z_{2}, \vec{r}_{2}, \alpha\right)\right] G_{\bar{q} q}\left(\vec{r}_{2}, z_{2} ; \vec{r}_{1}, z_{1}\right),
$$


with the boundary condition

$$
\left.G_{\bar{q} q}\left(\vec{r}_{2}, z_{2} ; \vec{r}_{1}, z_{1}\right)\right|_{z_{2}=z_{1}}=\delta^{2}\left(\vec{r}_{1}-\vec{r}_{2}\right) .
$$

In Eq. (2.8) $v$ is the photon energy and the Laplacian $\Delta_{r}$ acts on the coordinate $r$.

Studying propagation of a ${ }^{-} q q$ in vacuum the LC potential $V_{q q}\left(z_{2}, \vec{r}_{2}, \alpha\right)$ in (2.8) contains only the real part, which is responsible for the interaction between the $q$ and ${ }^{-} q$. For the oscillator form of this potential,

$$
\operatorname{Re} V_{\bar{q} q}\left(z_{2}, \vec{r}_{2}, \alpha\right)=\frac{a^{4}(\alpha) \vec{r}_{2}^{2}}{2 v \alpha(1-\alpha)}
$$

one can obtain the analytical solution of the evolution equation (2.8) represented by the harmonic oscillator Green function [15],

$$
\begin{array}{r}
G_{\bar{q} q}\left(\vec{r}_{2}, z_{2} ; \vec{r}_{1}, z_{1}\right)=\frac{a^{2}(\alpha)}{2 \pi i \sin (\omega \Delta z)} \exp \left\{\frac{i a^{2}(\alpha)}{\sin (\omega \Delta z)}\left[\left(r_{1}^{2}+r_{2}^{2}\right) \cos (\omega \Delta z)-2 \vec{r}_{1} \cdot \vec{r}_{2}\right]\right\} \\
\times \exp \left[-\frac{i \varepsilon^{2} \Delta z}{2 v \alpha(1-\alpha)}\right],
\end{array}
$$

where $\Delta z=z_{2}-z_{1}$ and

$$
\omega=\frac{a^{2}(\alpha)}{v \alpha(1-\alpha)} .
$$

The shape of the function $a(\alpha)$ in Eq. 2.10) is presented and discussed in ref. [5].

Matrix element (2.2) contains the LC wave function squared, which has the following form for $\mathrm{T}$ and $\mathrm{L}$ polarizations in the limit of vanishing interaction between ${ }^{-} q$ and $q$,

$$
\begin{gathered}
\left|\Psi_{\bar{q} q}^{T}\left(\vec{r}, \alpha, Q^{2}\right)\right|^{2}=\frac{2 N_{C} \alpha_{e m}}{(2 \pi)^{2}} \sum_{f=1}^{N_{f}} Z_{f}^{2}\left[m_{f}^{2} K_{0}(\varepsilon, r)^{2}+\left[\alpha^{2}+(1-\alpha)^{2}\right] \varepsilon^{2} K_{1}(\varepsilon r)^{2}\right], \\
\left|\Psi_{\bar{q} q}^{L}\left(\vec{r}, \alpha, Q^{2}\right)\right|^{2}=\frac{8 N_{C} \alpha_{e m}}{(2 \pi)^{2}} \sum_{f=1}^{N_{f}} Z_{f}^{2} Q^{2} \alpha^{2}(1-\alpha)^{2} K_{0}(\varepsilon r)^{2},
\end{gathered}
$$

where $K_{0}$ and $K_{1}$ are the modified Bessel functions.

The effects of higher Fock states $\left|{ }^{-} q q\right\rangle,\left|{ }^{-} q q G\right\rangle,\left|{ }^{-} q q 2 G\right\rangle, \ldots$ are implicitly incorporated into the energy dependence of the dipole cross section $\sigma_{\bar{q} q}(\vec{r}, s)$ naturally included in realistic KST parametrization Eq. (2.3).

Investigating DIS on nuclear targets, the corresponding formula for NS, $\Delta \sigma_{t o t}\left(x_{B j}, Q^{2}\right)=$ $\Delta \sigma_{t o t}\left({ }^{-} q q\right)$, representing the shadowing correction for the lowest ${ }^{-} q q$ Fock state, has the following form [7]

$$
\Delta \sigma_{t o t}\left(x_{B j}, Q^{2}\right)=\frac{1}{2} \operatorname{Re} \int d^{2} b \int_{-\infty}^{\infty} d z_{1} \rho_{A}\left(b, z_{1}\right) \int_{z_{1}}^{\infty} d z_{2} \rho_{A}\left(b, z_{2}\right) \int_{0}^{1} d \alpha A\left(z_{1}, z_{2}, \alpha\right),
$$

with

$$
A\left(z_{1}, z_{2}, \alpha\right)=\int d^{2} r_{2} \Psi_{\bar{q} q}^{*}\left(\vec{r}_{2}, \alpha, Q^{2}\right) \sigma_{\bar{q} q}\left(r_{2}, s\right) \int d^{2} r_{1} G_{\bar{q} q}\left(\vec{r}_{2}, z_{2} ; \vec{r}_{1}, z_{1}\right) \sigma_{\bar{q} q}\left(r_{1}, s\right) \Psi_{\bar{q} q}\left(\vec{r}_{1}, \alpha, Q^{2}\right) .
$$




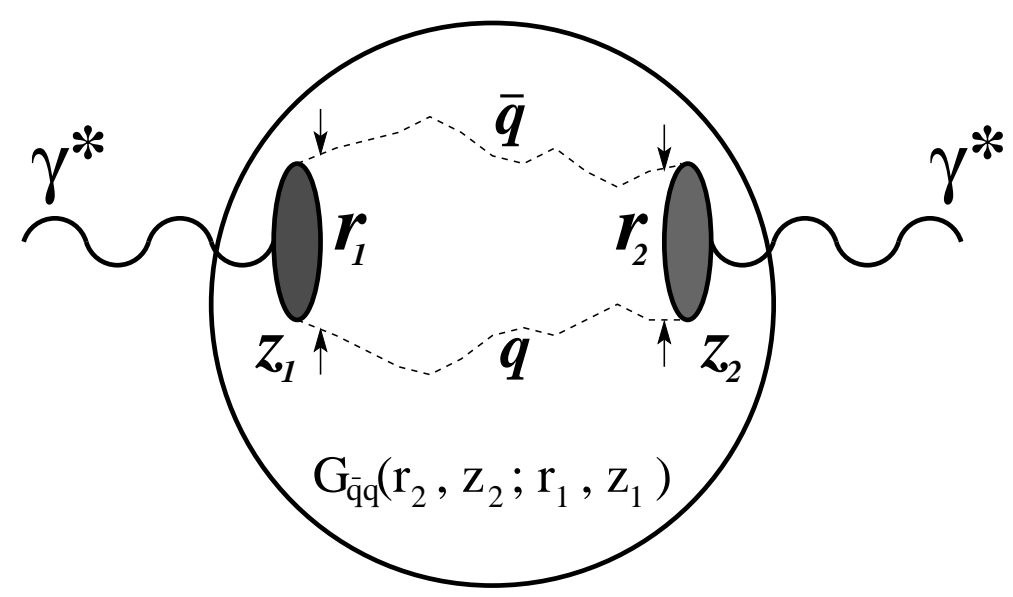

Figure 1: A cartoon for the shadowing term $\Delta \sigma_{t o t}\left(x_{B j}, Q^{2}\right)=\Delta \sigma_{t o t}(\bar{q} q)$ in (2.15). Propagation of the $\bar{q} q$ pair through the nucleus is described by the Green function $G_{\bar{q} q}\left(\vec{r}_{2}, z_{2} ; \vec{r}_{1}, z_{1}\right)$, which results from the summation over different paths of the $\bar{q} q$ pair.

In Eq. 2.15) $\rho_{A}(b, z)$ represents the nuclear density function defined at the point with longitudinal coordinate $z$ and impact parameter $\vec{b}$.

The shadowing term $\Delta \sigma_{t o t}\left(x_{B j}, Q^{2}\right)=\Delta \sigma_{t o t}\left({ }^{-} q q\right)$ in (2.1 $)$ is illustrated in Fig $\square$. At the point $\bar{x}$ the initial photon diffractively produces the ${ }^{-} q q$ pair $\left(\gamma N \rightarrow{ }^{-} q q N\right)$ with transverse separation $\vec{r}$. The - $q q$ pair then propagates through the nucleus along arbitrary curved trajectories, which are summed over, and arrives at the point $z_{2}$ with transverse separation $\vec{r}_{2}$. The initial and final separations are controlled by the LC wave function of the ${ }^{-} q q$ Fock component of the photon $\Psi_{\bar{q} q}\left(\vec{r}, \alpha, Q^{2}\right)$. During propagation through the nucleus the ${ }^{-} q q$ pair interacts with bound nucleons via the dipole cross section $\sigma_{\bar{q} q}(r, s)$, which depends on the local transverse separation $\vec{r}$. The Green function $G_{\bar{q} q}\left(\vec{r}_{2}, z_{2} ; \vec{r}_{1}, z_{1}\right)$ describes the propagation of the ${ }^{-} q q$ pair from $\bar{q}$ to $z_{2}$.

Describing propagation of the ${ }^{-} q q$ pair in nuclear medium the Green function $G_{q q}\left(\vec{r}_{2}, z_{2} ; \vec{r}_{1}, z_{1}\right)$ satisfies again the time-dependent two-dimensional Schrödinger equation (2.8). However, the potential in this case acquires in addition also the imaginary part responsible for attenuation of the ${ }^{-} q q$ photon fluctuation in the medium and has the following form

$$
\operatorname{Im} V_{\bar{q} q}\left(z_{2}, \vec{r}, \alpha\right)=-\frac{\sigma_{\bar{q} q}(\vec{r}, s)}{2} \rho_{A}\left(b, z_{2}\right) .
$$

As we already mentioned above the analytical solution of Eq. (2.8) is known only for the harmonic oscillator potential $V_{\bar{q} q}(r) \propto r^{2}$, i.e.

$$
\sigma_{\bar{q} q}(r, s)=C(s) r^{2}
$$

and uniform nuclear density

$$
\rho_{A}(b, z)=\rho_{0} \Theta\left(R_{A}^{2}-b^{2}-z^{2}\right)
$$

in Eq. (2.17) for the imaginary part of the LC potential. 
In this case the solution of Eq. (2.8) has the same form as Eq. (2.11), expect that one should replace $\omega \Longrightarrow \Omega$ and $a^{2}(\alpha) \Longrightarrow b(\alpha)$, where

$$
\Omega=\frac{b(\alpha)}{v \alpha(1-\alpha)}=\frac{\sqrt{a^{4}(\alpha)-i \rho_{A}(b, z) v \alpha(1-\alpha) C(s)}}{v \alpha(1-\alpha)} .
$$

Determination of the energy dependent factor $C(s)$ in Eq. (2.18) and the mean nuclear density $\rho_{0}$ in Eq. (2.19) can be realized by the procedure described in [ [ 7 , 5].

In the general case when there is no restrictions for $l_{c}$, if $l_{c} \sim R_{A}$ one has to take into account the variation of the transverse size $r$ during propagation of the ${ }^{-} q q$ pair through the nucleus. The overall total photoabsorption cross section on a nucleus $\sigma^{\gamma^{*} A}=\sigma_{T}^{\gamma^{*} A}+\varepsilon^{\prime} \sigma_{L}^{\gamma^{*} A}$, assuming that the photon polarization $\varepsilon^{\prime}=1$. If one takes into account only ${ }^{-} q q$ Fock component of the photon the full expression after summation over all flavors, colors, helicities and spin states has the following form [16]

$$
\begin{aligned}
& \sigma^{\gamma^{*} A}\left(x_{B j}, Q^{2}\right)=A \sigma^{\gamma^{*} N}\left(x_{B j}, Q^{2}\right)-\Delta \sigma\left(x_{B j}, Q^{2}\right) \\
&= A \int d^{2} r \int_{0}^{1} d \alpha \sigma_{\bar{q} q}(r, s)\left(\left|\Psi_{\bar{q} q}^{T}\left(\vec{r}, \alpha, Q^{2}\right)\right|^{2}+\left|\Psi_{\bar{q} q}^{L}\left(\vec{r}, \alpha, Q^{2}\right)\right|^{2}\right) \\
&-\frac{N_{C} \alpha_{e m}}{(2 \pi)^{2}} \sum_{f=1}^{N_{f}} Z_{f}^{2} \Re e \int d^{2} b \int_{-\infty}^{\infty} d z_{1} \int_{z_{1}}^{\infty} d z_{2} \int_{0}^{1} d \alpha \int d^{2} r_{1} \int d^{2} r_{2} \\
& \times \rho_{A}\left(b, z_{1}\right) \rho_{A}\left(b, z_{2}\right) \sigma_{\bar{q} q}\left(r_{2}, s\right) \sigma_{\bar{q} q}\left(r_{1}, s\right)\left\{\left[\alpha^{2}+(1-\alpha)^{2}\right] \varepsilon^{2} \frac{\vec{r}_{1} \cdot \vec{r}_{2}}{r_{1} r_{2}} K_{1}\left(\varepsilon r_{1}\right) K_{1}\left(\varepsilon r_{2}\right)\right. \\
&\left.+\quad\left[m_{f}^{2}+4 Q^{2} \alpha^{2}(1-\alpha)^{2}\right] K_{0}\left(\varepsilon r_{1}\right) K_{0}\left(\varepsilon r_{2}\right)\right\} G_{\bar{q} q}\left(\vec{r}_{2}, z_{2} ; \vec{r}_{1}, z_{1}\right) .
\end{aligned}
$$

The shape of $\left|\Psi_{\bar{q} q}^{T, L}\left(\vec{r}, \alpha, Q^{2}\right)\right|^{2}$ is given by Eqs. (2.13) and (2.14), respectively.

In the high energy limit, $l_{c} \gg R_{A}$, the transverse separation $r$ between $-q$ and $q$ does not vary during propagation through the nucleus (Lorentz time dilation). Consequently, the kinetic term in Eq. (2.8) can be neglected and the Green function reads

$$
\left.G_{\bar{q} q}\left(b ; \vec{r}_{2}, z_{2} ; \vec{r}_{1}, z_{1}\right)\right|_{v \rightarrow \infty}=\delta\left(\vec{r}_{2}-\vec{r}_{1}\right) \exp \left[-\frac{1}{2} \sigma_{\bar{q} q}\left(r_{2}, s\right) \int_{z_{1}}^{z_{2}} d z \rho_{A}(b, z)\right] .
$$

After substitution of the expression (2.22) into Eq. (2.21), one arrives at the following results:

$$
\begin{gathered}
\sigma_{n p t}^{\gamma^{*} A}\left(x_{B j}, Q^{2}\right)=2 \int d^{2} b \int d^{2} r \int_{0}^{1} d \alpha\left\{1-\exp \left[-\frac{1}{2} \sigma_{\bar{q} q}(r, s) T_{A}(b)\right]\right\} \\
\times \frac{2 N_{C} \alpha_{e m}}{(2 \pi)^{2}} \sum_{f=1}^{N_{f}} Z_{f}^{2}\left\{\left[\alpha^{2}+(1-\alpha)^{2}\right] K_{1}(\varepsilon r)^{2}+\left[m_{f}^{2}+4 Q^{2} \alpha^{2}(1-\alpha)^{2}\right] K_{0}^{2}(\varepsilon r)\right\},
\end{gathered}
$$

where

$$
T_{A}(b)=\int_{-\infty}^{\infty} d z \rho_{A}(b, z)
$$


is the nuclear thickness calculated with the realistic Wood-Saxon form of the nuclear density with parameters taken from [17].

At photon polarization parameter $\varepsilon^{\prime}=1$ the structure function ratio $F_{2}^{A}\left(x_{B j}, Q^{2}\right) / F_{2}^{N}\left(x_{B j}, Q^{2}\right)$ can be expressed via ratio of the total photoabsorption cross sections

$$
\frac{F_{2}^{A}\left(x_{B j}, Q^{2}\right)}{F_{2}^{N}\left(x_{B j}, Q^{2}\right)}=\frac{\sigma_{T}^{\gamma^{*} A}\left(x_{B j}, Q^{2}\right)+\sigma_{L}^{\gamma^{*} A}\left(x_{B j}, Q^{2}\right)}{\sigma_{T}^{\gamma^{*} N}\left(x_{B j}, Q^{2}\right)+\sigma_{L}^{\gamma^{*} N}\left(x_{B j}, Q^{2}\right)},
$$

where the numerator on right-hand side (r.h.s.) is given by Eq. (2.21), whereas denominator can be expressed as the first term of Eq. (2.21) divided by the mass number $A$.

The nonperturbative ${ }^{-} q-q$ interaction is included by replacements $K_{\gamma}(\varepsilon r) / 2 \pi \Longrightarrow \Phi_{0}(\varepsilon, r, \lambda)$ and $K_{1}(\varepsilon r) / 2 \pi \Longrightarrow \Phi_{1}(\varepsilon, r, \lambda)$ in all perturbative expressions. The corresponding functions $\Phi_{0,1}$ and parameter $\lambda$ are defined in ref. [6].

We solve the evolution equation for the Green function, Eq. (2.8), numerically using algorithm from ref. [5]. Such an exact solution is performed for realistic KST parametrization of the dipole cross section (2.3) and nuclear density function in the realistic Wood-Saxon form with parameters taken from ref. [17].

Finally we would like to emphasize (see also the next section) that the ${ }^{-} q q$ Fock component of the photon represents the higher twist shadowing correction [ $\llbracket$, and vanishes at large quark masses as $1 / m_{f}^{2}$. This does not happen for higher Fock states containing gluons, which lead to GS. Therefore GS represents the leading twist shadowing correction [6]. Moreover, a steep energy dependence of the dipole cross section $\sigma_{\bar{q} q}(r, s)$ (see Eq. (2.3) ) especially at smaller dipole sizes $r$ causes a steep energy rise of both corrections.

\section{Gluon shadowing}

Investigating nuclear effects at small $x_{B j} \lesssim 0.01$, the higher Fock states of the photon containing gluons also contribute to NS. Because of a shorter coherence time (lifetime) of these fluctuations, GS will be dominated at higher energies, i.e. at smaller $x_{B j}$ - values than NS coming from the lowest $\mid{ }^{-} q q>$ Fock component. However, no data for GS are available and one should rely on calculations.

NS for $\left|{ }^{-} q q\right\rangle$ Fock component of the photon is dominated by T photon polarizations, because the corresponding photoabsorption cross section is scanned at larger dipole sizes than for the L photon polarization. The transverse ${ }^{-} q q$ separation is controlled by the distribution amplitude Eq. (2.5), with the mean value given by Eq. (2.7). Contributions of large size dipoles come from the asymmetric - $q q$ fluctuations of the virtual photon, when the quark and antiquark in the photon carry a very large $(\alpha \rightarrow 1)$ and a very small fraction $(\alpha \rightarrow 0)$ of the photon momentum, and vice versa. The $\mathrm{LC}$ wave function for $\mathrm{L}$ photons (2.14) contains a term $\alpha^{2}(1-\alpha)^{2}$, which makes considerably smaller the contribution from asymmetric ${ }^{-} q q$ configurations than for T photons (see Eq. (2.13)). Consequently, in contrast to $\mathrm{T}$ photons, all ${ }^{-} q q$ dipoles from $\mathrm{L}$ photons have a size squared $\propto 1 / \mathcal{Q}$ and the doublescattering term vanishes as $\propto 1 / Q^{4}$. The leading-twist contribution for the shadowing of $\mathrm{L}$ photons arises from the $\left|{ }^{-} q q G\right\rangle$ Fock component of the photon because the gluon can propagate relatively far from the ${ }^{-} q q$ pair, although the ${ }^{-} q-q$ separation is of the order $1 / \mathcal{Q}$ After radiation of the gluon 
the pair is in an octet state and consequently the $\left|{ }^{-} q q G\right\rangle$ state represents a $G G$ dipole. Then the corresponding correction to the $\mathrm{L}$ cross section is just GS.

Interpretation of GS is reference frame dependent. In the infinite momentum frame this phenomenon looks analogical as gluon-gluon fusion. Within a parton model interpretation, the gluon clouds of nucleons which have the same impact parameter overlap at small $x_{B j}$ in longitudinal direction. This allows gluons originated from different nucleons to fuse leading to a gluon density which is not proportional to the density of nucleons any more. This is GS.

In the rest frame of the nucleus phenomenon of GS corresponds to the process of gluon radiation and shadowing corrections related to multiple interactions of the radiated gluons in the nuclear medium. This is a coherence phenomenon known as the Landau-Pomeranchuk effect, namely the suppression of bremsstrahlung by interference of radiation from different scattering centers. It demands a sufficiently long coherence time of radiation, a condition equivalent to demanding a small Bjorken $x_{B j}$ in the parton model.

There are still very few numerical evaluations of GS in the literature, all done in the rest frame of the nucleus. GS can be identified as the shadowing correction to the $\mathrm{L}$ cross section coming from the $G G$ dipole representing $\left|{ }^{-} q q G\right\rangle$ Fock component of the photon. For evaluation of GS is important to know about the transverse size of this $G G$ dipole. This size has been extracted in ref. [6] from data for diffractive excitation of the incident hadrons to the states of large mass, the so called triple-Pomeron region. It was found that the mean dipole size of the $G G$ system (radius of propagation of the LC gluons) is rather small, $r_{0} \approx 0.3 \mathrm{fm}[18]$. It results in a not very strong onset of GS.

The smallness of the size of quark-gluon fluctuations has been incorporated via a nonperturbative LC potential in the Schrödinger equation for the Green function describing the propagation of a quark-gluon system. The strength of the potential was fixed by data on high mass $\left(M_{X}^{2}\right)$ diffraction $p p \rightarrow p X$ [6]. This approach allows to extend the methods of pQCD to the region of small $Q^{2} \lesssim Q_{0}^{2}=4 / r_{0}^{2}$. At higher $Q^{2} \gtrsim Q_{0}^{2}$ GS slowly (logarithmically) decreases in accordance with expectations based on the evolution equation [2].

We repeated the calculations [6] of the ratio of the gluon densities in nuclei and nucleon,

$$
R_{G}\left(x_{B j}, Q^{2}\right)=\frac{G_{A}\left(x_{B j}, Q^{2}\right)}{A G_{N}\left(x_{B j}, Q^{2}\right)} \approx 1-\frac{\Delta \sigma_{t o t}\left({ }^{-} q q G\right)}{\sigma_{t o t}^{\gamma^{*} A}},
$$

where $\Delta \sigma_{t o t}\left({ }^{-} q q G\right)$ is the inelastic correction to the total cross section ${ }_{t o t}^{{ }^{*} A}$ related to the creation of a $\left.{ }^{-} q q G\right\rangle$ intermediate Fock state. Further calculation details can be found in [\$] $]$. As an illustration of not very strong onset of GS, here we present $R_{G}\left(x_{B j}, Q^{2}\right)$, Eq. (3.1), for different nuclear thicknesses $T_{A}(b)$. Using an approximation of constant nuclear density (see Eq. (2.19)), $T_{A}(b)=\rho_{0} L$, where $L=2 \sqrt{R_{A}^{2}-b^{2}}$, the ratio $R_{G}\left(x_{B j}, Q^{2}\right)$ is also implicitly a function of $L$. An example for the calculated $L$-dependence of $R_{G}\left(x_{B j}, Q^{2}\right)$ at $Q^{2}=4 \mathrm{GeV}^{2}$ is depicted in Fig. 2 for different values of $x_{B j}$.

We calculated GS only for the lowest Fock component containing just one LC gluon. Inclusion of higher multigluon Fock components is still a challenge. However, their effect can be essentially taken into account by eikonalization of the calculated $R_{G}\left(x_{B j}, Q^{2}\right)$, i.e. the dipole cross section, 


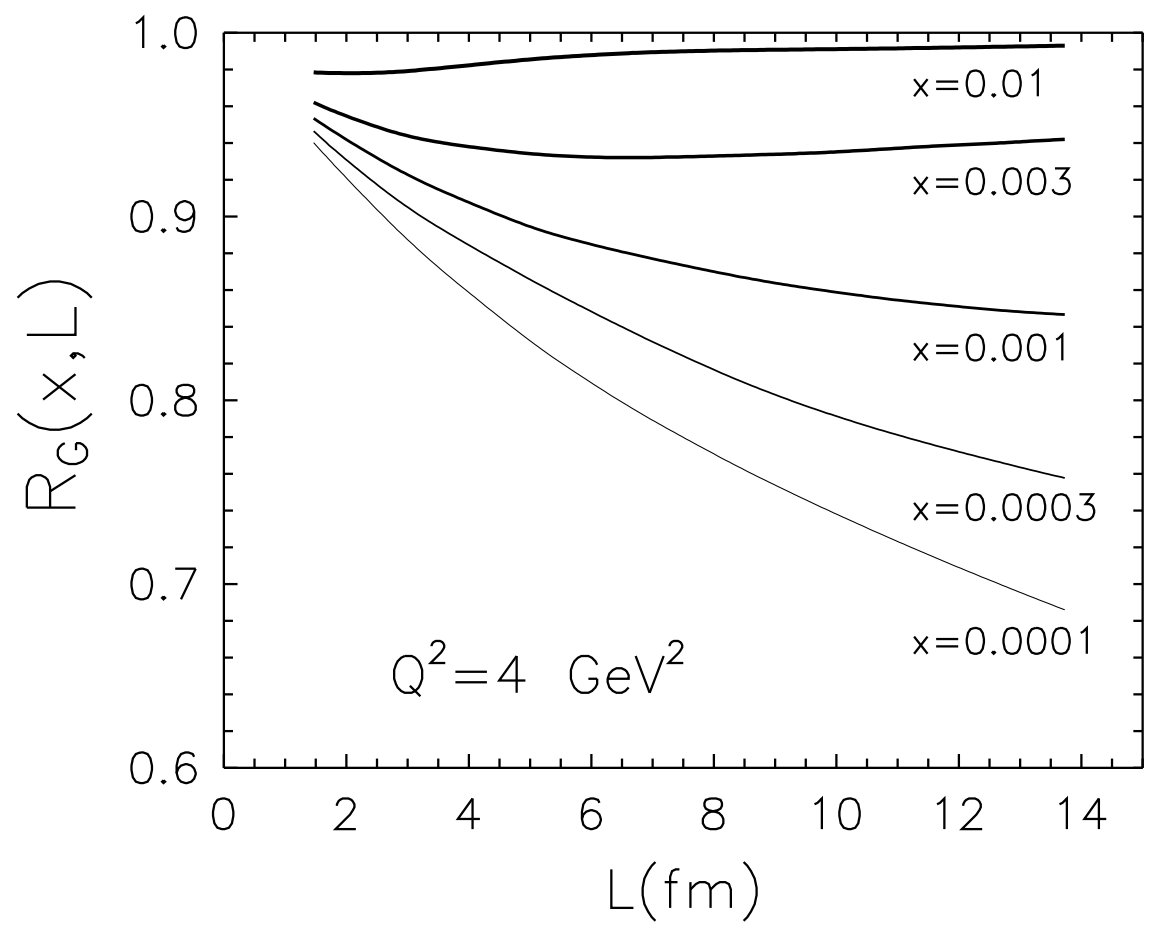

Figure 2: The ratio of the nucleus-to-nucleon gluon densities as function of the thickness of the nucleus, $L=T(b) / \rho_{0}$, at $Q^{2}=4 \mathrm{GeV}^{2}$ and different fixed values of $x_{B j}$. Figure is taken from ref. [1].

which is proportional to the gluon density at small separations, should be renormalized everywhere,

$$
\sigma_{\bar{q} q} \Rightarrow R_{G} \sigma_{\bar{q} q}
$$

According to Eq. (3.2) we will demonstrate that GS suppresses the total photoabsorption cross section on a nucleus $\sigma_{\text {tot }}^{\gamma^{*} A}\left(x_{B j}, Q^{2}\right)$. We expect a non-negligible effect of GS in the shadowing region of small $x_{B j} \lesssim(0.001 \div 0.01)$ and at small and medium values of $Q^{2} \sim 2 \div 3 \mathrm{GeV}^{2}$ corresponding to the kinematic range of available data.

\section{Numerical results}

Here we present the available data vs. realistic predictions for NS in DIS based on exact numerical solutions of the evolution equation for the Green function corresponding to the lowest - $q q$ Fock component of the photon. Such a comparison is performed for the shadowing region of small $x_{B j} \lesssim 0.01$.

We take into account also a contribution of GS which should increase overall nuclear suppression. The effects of GS are calculated for the lowest Fock component containing just one LC gluon. Inclusion of higher Fock components with more gluons is realized by eikonalization of the calculated $R_{G}\left(x_{B j}, Q^{2}\right.$ ), i.e using renormalization (3.2).

For a numerical solution of the Schrödinger equation for the Green function we adopt an algorithm from ref. [5]. Because available data from the E665 [19] and NMC [20] collaborations 


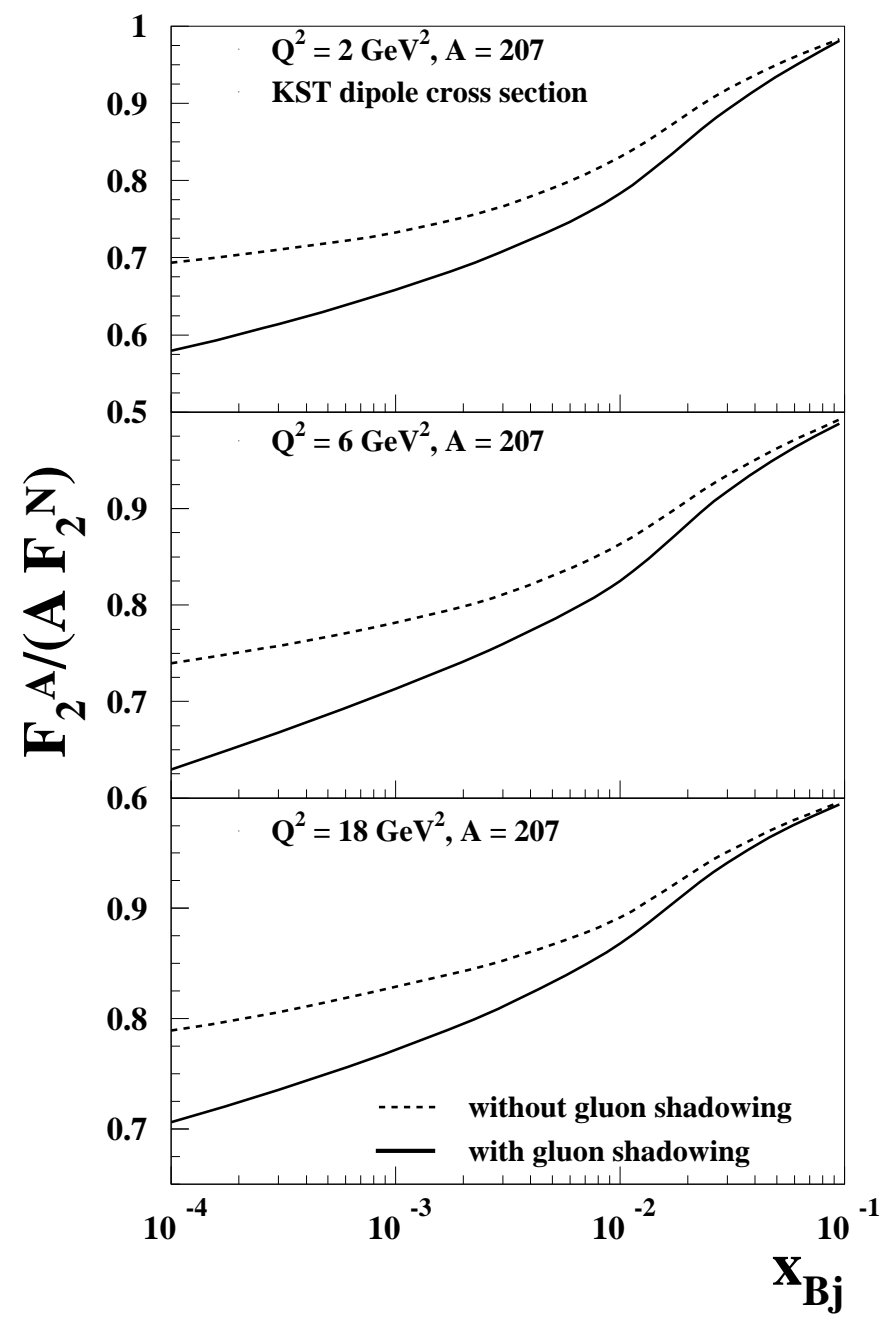

Figure 3: Nuclear shadowing for lead. Calculations correspond to exact numerical solution of the evolution equation for the Green function, $G_{\bar{q} q}\left(\vec{r}_{2}, z_{2} ; \vec{r}_{1}, z_{1}\right)$, for the lowest Fock component of the photon using KST [6] parametrization of the dipole cross section and realistic nuclear density function of the Woods-Saxon form [17]. The solid and dashed curves represent the predictions calculated with and without contribution of gluon shadowing, respectively.

cover the region of small and medium values of $Q^{2} \lesssim 4 \mathrm{GeV}^{2}$ we use the KST parametrization of the dipole cross section [6] which is valid down to the limit of real photoproduction.

Performing a numerical solution of the evolution equation for the Green function, the imaginary part of the LC potential (2.17) contains the corresponding KST dipole cross section as well. The nuclear density function $\rho_{A}(b, z)$ is taken in the realistic Wood-Saxon form with parameters from ref. [17]. Because available data from the E665 and NMC collaborations correspond to very small values of $Q^{2} \lesssim 1 \mathrm{GeV}^{2}$ at small $x_{B j} \lesssim 0.004$, the nonperturbative interaction effects between ${ }^{-} q$ and $q$ are included explicitly via the real part of the LC potential of the form (2.1)).

Effects of NS are studied via $x_{B j}$ - behavior of the ratio (2.25) divided by the mass number $A$. Firstly we present NS for the lead target in Fig. 3 at different fixed values of $Q^{2}$. The solid and dashed curves represent the predictions obtained with and without contribution of GS, respectively. 
One can see that as a manifestation of a shorter $l_{c}$ for higher Fock states, the onset of GS happens at smaller $x_{B j}$ than the quark shadowing. Fig. B 3 clearly demonstrates not very strong onset of GS in the range of $x_{B j} \in(0.01,0.0001)$ where the most of available data exist. Besides the effects of GS are stronger at smaller $Q^{2}$ because corresponding Fock fluctuations of the photon have a larger transverse size.
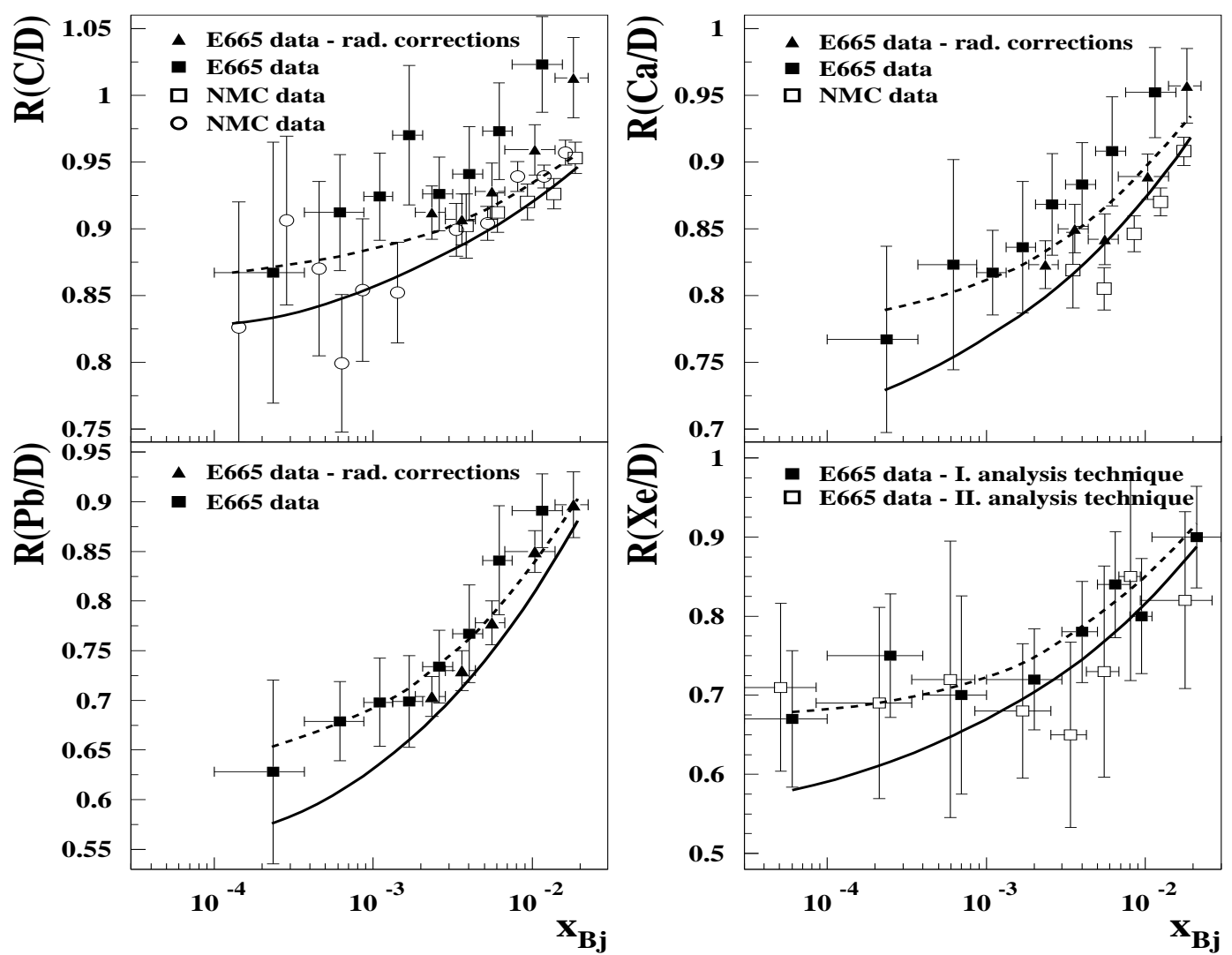

Figure 4: Comparison of the model with experimental data from the E665 [19] and NMC [20] collaborations. Calculations correspond to exact numerical solution of the evolution equation for the Green function, $G_{\bar{q} q}\left(\vec{r}_{2}, z_{2} ; \vec{r}_{1}, z_{1}\right)$, for the lowest Fock component of the photon using KST [0] parametrization of the dipole cross section and realistic nuclear density function of the WoodsSaxon form [17]. The solid and dashed curves are calculated with and without contribution of gluon shadowing, respectively.

Saturation of NS at low $x_{B j} \lesssim 10^{-4}$ at the level given by Eq. (2.23) is realized only for energy independent dipole cross section (see parametrization (2.18)). However, it is not so for the realistic energy-dependent KST parametrization, Eq. (2.3).

In Fig. 7 we present a comparison of the model predictions with data from the E665 [19] and NMC [20] collaborations. Fig. shows a quite reasonable agreement with experimental data in spite of absence of any free parameters in the model. One can see that the effect of GS produces an additional NS, which rises with the mass number $A$. Fig. \# Also demonstrates a non-negligibility of GS already in the region of $x_{B j} \lesssim 0.001 \div 0.01$. Very large error bars especially at small $x_{B j} \sim 10^{-4}$ do not allow to investigate separately the effect of GS. Therefore more accurate new data on NS in 
DIS at small $x_{B j}$ are very important for the further exploratory study of nuclear modification of the structure functions and also GS.

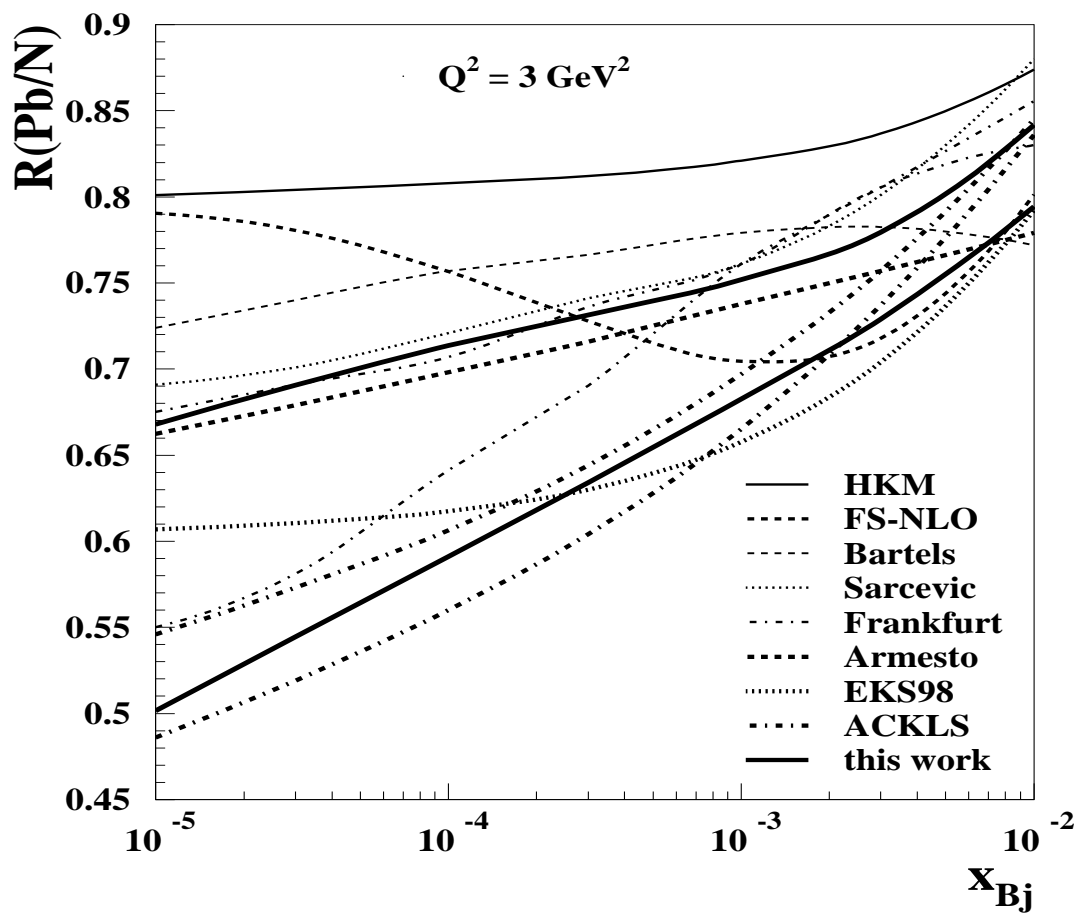

Figure 5: Comparison of the model predictions for the ratio $\mathrm{Pb}$ /nucleon obtained without (upper thick solid line) and with GS (lower thick solid line) with other models, versus $x_{B j}$ at fixed $Q^{2}=$ $3 \mathrm{GeV}^{2}$. HKM are the results from [24], FS-NLO from [22], Bartels from [28], Sarcevic from [26], Frankfurt from [2]] $\left(Q^{2}=4 \mathrm{GeV}^{2}\right)$, Armesto from [25], EKS98 from [23] and ACKLS from [27].

Finally, Fig. 5 represents a comparison of the model predictions for NS with the results from other models at $Q^{2}=3 \mathrm{GeV}^{2}$ (except the results of ref. [21] which are at $Q^{2}=4 \mathrm{GeV}^{2}$ ). At small $x_{B j}=10^{-5}$ we predict quite large effects of GS (compare upper and lower thick solid lines). Note that the difference between models rises towards small values of $x_{B j}$ following from a different treatment of various nuclear effects.

The models presented in Fig. 5 can be divided into several groups:

i) models where parton distribution functions (PDF's) has been determined from data using Dokshitzer-Gribov-Lipatov-Altareli-Parisi (DGLAP) evolution equation

Here the nuclear PDF's have been parametrized performing next to leading order (NLO) [2]] or leading order (LO) [23, 24] global analysis of nuclear DIS and Drell-Yan data.

ii) models based on Glauber-like rescatterings

Here the model in ref. [25] is based on an application of a saturating ansatz for the total $\gamma^{*}$-nucleon cross section in the proton. This ansatz is then extended to the nuclear case by its introduction in a Glauber expression. Within the model from [26] a Glauber ansatz provides with the initial condition for DGLAP evolution.

iii) models based on Gribov inelastic shadowing

Here in ref. [27] nuclear structure functions are studied using relation with diffraction on nucleons 
which arises from Gribov's reggeon calculus. The model presented in [21] employs again some parametrization of hard diffraction at the scale $Q_{0}^{2}$, which gives nuclear shadowing through Gribov's reggeon calculus similarly as in ref. [27]. Then nuclear suppression calculated at $Q_{0}^{2}$ is used as initial condition for DGLAP evolution.

iv) models based on high-density $Q C D$

Here the model in ref. [28] is based on numerical solution of a non-linear equation for small- $x_{B j}$ evolution and on application of this equation for the case of nuclear targets.

Fig. 5 shows large differences in predictions of NS at small $x_{B j}$ among different models. It gives a stimulation to obtain new more accurate data on nuclear structure functions by lepton-ion collider planned at RHIC [29]. It can help us to discriminate among different models.

\section{Summary and conclusions}

We present a short review of the color dipole approach based on the LC QCD Green function formalism, which naturally incorporates the interference effects of CT and CL. Within this approach [7, 4, 5] we study NS in DIS at small Bjorken $x_{B j}$.

Calculations of NS corresponding to ${ }^{-} q q$ component of the virtual photon are based on an exact numerical solution of the evolution equation for the Green function. It allows to use realistic parametrizations of the dipole cross section (GBW [10] and KST [6]) and realistic nuclear density function of the Woods-Saxon form [17].

Because available data from the shadowing region of $x_{B j} \lesssim 0.01$ coming mostly from the E665 and NMC collaborations cover only small and medium values of $Q^{2} \lesssim 4 \mathrm{GeV}^{2}$, we prefer KST parametrization [6] of the dipole cross section. On the other hand the data obtained at a lower part of the $x_{B j}$ - kinematic interval correspond to very low values of $Q^{2}<1 \mathrm{GeV}^{2}$ (nonperturbative region). For this reason we include explicitly the nonperturbative interaction effects between ${ }^{-} q$ and $q$ taking into account the real part of the LC potential $V_{\bar{q} q}(2.10)$ in the time-dependent twodimensional Schrödinger equation (2.8)

In order to compare the realistic calculations with data on NS, the effects of GS are taken into account. The same path integral technique [6] is applied in this case. GS was calculated only for the lowest Fock component, $\left|{ }^{-} q q G\right\rangle$. Effect of higher Fock components containing more gluons was essentially taken into account by eikonalization of the calculated $R_{G}\left(x_{B j}, Q^{2}\right)$ using renormalization (3.2). We demonstrate that the onset of GS starts to be effective at $x_{B j} \sim 0.01$. It rises towards small $x_{B j}$ because higher Fock components with more gluons having shorter coherence time will contribute to overall NS. Such a situation is illustrated in Fig. 3 .

Performing numerical calculations, we find that our model is in reasonable agreement with existing experimental data (see Fig. (7). Large error bars and incompatibility of the experimental results from the E665 and NMC collaborations do not allow to study separately the effect of GS. Therefore more accurate new data on NS in DIS off nuclei at still smaller $x_{B j} \lesssim 10^{-5}$ are very important for the further exploratory study of GS effects.

Comparison among various models shows large differences for the $\mathrm{Pb} /$ nucleon ratio of the structure functions at $x_{B j}=10^{-5}$ and $Q^{2}=3 \mathrm{GeV}^{2}$ (see Fig. 5). It has a large impact on the calculation of high- $p_{T}$ particle spectra in nuclear collisions at RHIC and LHC. Such large differences 
at small $x_{B j}$ among different models should be testable by the new more precise data on nuclear structure functions which can be obtained by lepton-ion collider planned at RHIC [29].

Acknowledgments: This work has been supported in part by the Slovak Funding Agency, Grant No. 2/7058/27.

\section{References}

[1] O.V. Kancheli, Sov. Phys. JETP Lett. 18 (1973) 274; V.N. Gribov, E.M. Levin and M.G. Ryskin, Phys. Rept. 100 (1983) 1.

[2] A.H. Mueller and J. Qiu, Nucl. Phys. B 268 (1986) 427.

[3] N.N. Nikolaev and B.G. Zakharov, Z. Phys. C 49 (1991) 607.

[4] B.Z. Kopeliovich, J. Raufeisen and A.V. Tarasov, Phys. Rev. C 62 (2000) 035204.

[5] J. Nemchik, Phys. Rev. C 68 (2003) 035206.

[6] B.Z. Kopeliovich, A. Schäfer and A.V. Tarasov, Phys. Rev. D 62 (2000) 054022.

[7] B.Z. Kopeliovich, J. Raufeisen and A.V. Tarasov, Phys. Lett. B 440 (1998) 151.

[8] A.B. Zamolodchikov, B.Z. Kopeliovich and L.I. Lapidus, Sov. Phys. JETP Lett. 33 (1981) 595.

[9] G. Bertsch, S.J. Brodsky, A.S. Goldhaber and J.F. Gunion, Phys. Rev. Lett. 47 (1981) 297.

[10] K. Golec-Biernat and M. Wüsthoff, Phys. Rev. D 59 (1999) 014017; Phys. Rev. D 60 (1999) 114023.

[11] B.Z. Kopeliovich, J. Nemchik, A. Schaefer and A.V. Tarasov, Phys. Rev. C 65 (2002) 035201.

[12] Review of Particle Physics, R.M. Barnett et al., Phys. Rev. D 54 (1996) 191.

[13] J.B. Kogut and D.E. Soper, Phys. Rev. D 1 (1970) 2901.

[14] J.M. Bjorken, J.B. Kogut and D.E. Soper, Phys. Rev. D 3 (1971) 1382.

[15] R.P. Feynman and A.R. Gibbs, Quantum Mechanics and Path Integrals, McGraw-Hill, New York, 1965.

[16] B.G. Zakharov, Phys. Atom. Nucl. 61 (1998) 838.

[17] H.De Vries, C.W.De Jager and C.De Vries, Atomic Data and Nucl. Data Tables 36 (1987) 469.

[18] B.Z. Kopeliovich, I.K. Potashnikova, B. Povh and E. Predazzi, Phys. Rev. Lett. 85 (2000) 507.

[19] E665 Collaboration, M.R. Adams et al., Z. Phys. C67 (1995) 403;

E665 Collaboration, M.R. Adams et al., Phys. Rev. Lett. 68 (1992) 3266.

[20] NMC Collaboration, P. Amaudruz et al., Nucl. Phys. B441 (1995) 3;

NMC Collaboration, M. Arneodo et al., Nucl. Phys. B441 (1995) 12.

[21] L. Frankfurt, V. Guzey, M. McDermott and M. Strikman, JHEP 0202 (2002) 027;

L. Frankfurt, V. Guzey and M. Strikman, Phys. Rev. D71 (2005) 054001

[22] D. de Florian, R. Sassot, Phys. Rev. D69 (2004) 074208.

[23] K.J. Eskola, V.J. Kolhinen and P.V. Ruuskanen, Nucl. Phys. B535 (1998) 351;

K.J. Eskola, V.J. Kolhinen and C.A. Salgado, Eur. Phys. J. C9 (1999) 61.

[24] M. Hirai, S. Kumano and M. Miyama, Phys. Rev. D64 (2001) 034003. 
[25] N. Armesto, Eur. Phys. J. C26 (2002) 35.

[26] Z. Huang, H.J. Lu and I. Sarcevic, Nucl. Phys. A637 (1998) 79.

[27] N. Armesto, A. Capella, A.B. Kaidalov, J.López-Albacete and C.A. Salgado, Eur. Phys. J. C29 (2003) 531.

[28] J. Bartels, E. Gotsman, E.M. Levin, M. Lublinsky and U. Maor, Phys. Rev. D68 (2003) 054008.

[29] R.G. Milner, in Proceedings of the XII International Workshop on DIS, 14-18 April 2004, Štrbské Pleso, Slovakia, 173-182. 